(c) 2018 IEEE. Personal use of this material is permitted. Permission from IEEE must be obtained for all other uses, in any current or future media, including reprinting/republishing this material for advertising or promotional purposes, creating new collective works, for resale or redistribution to servers or lists, or reuse of any copyrighted component of this work in other works.

DOI: 10.1109/CAMA.2018.8530536 


\title{
Characterization of a mm-Wave Automotive Radar with a Low-RCS MIMO Antenna System
}

\author{
Claudia Vasanelli ${ }^{1}$, Klaus Baur ${ }^{2}$, Martin Nezadal ${ }^{2}$, Heiko Gulan ${ }^{2}$, Maximilian Steiner ${ }^{1}$, and Christian Waldschmidt ${ }^{1}$ \\ ${ }^{1}$ Ulm University, Institute of Microwave Engineering, $89081 \mathrm{Ulm}$, Germany \\ \{claudia.vasanelli, maximilian.steiner, christian.waldschmidt\}@uni-ulm.de \\ ${ }^{2}$ Robert Bosch GmbH, Chassis Systems Control, 71229 Leonberg, Germany \\ \{klaus.baur, martin.nezadal, heiko.gulan\}@de.bosch.com
}

\begin{abstract}
Millimeter-wave (mm-wave) automotive radars are typically installed behind the car fascia. However, the placement behind the bumper can cause multiple reflections, which may lead to a wrong estimation of the direction of arrival (DoA) of the target. The use of low-RCS (radar cross-section) antennas can mitigate them. This work presents the characterization of a mmwave frequency-modulated continuous-wave (FMCW) MIMO (multiple-input multiple-output) radar equipped with a low-RCS antenna systems. The measurement results demonstrate that such an antenna configuration can reduce the angular error in the DoA estimation and increase the robustness of the system.
\end{abstract}

\section{INTRODUCTION}

The car industry employs mm-wave radars, working between $76 \mathrm{GHz}$ and $81 \mathrm{GHz}$, for driver assistance systems [1]. The radars can measure the distance and the velocity of the surrounding targets on the street. Moreover, if they are equipped with more than one channel they can also provide angular information of the target by estimating the DoA [2].

Thanks to the small free-space wavelength, the sensors have compact dimensions, and thus, they can be easily integrated in the vehicle. The preferred solution by car manufacturers is the hidden integration of the sensors behind the vehicle fascia, i.e., the front and rear bumpers. However, a car bumper is not designed to facilitate the placement of a mm-wave radar [3]. It can indeed significantly degrade the sensor performance. The bumper is a complex multilayer structure. From an electromagnetic point of view, the external coating is responsible for a strong reflection of the impinging electromagnetic waves coming from the radar, since it often contains metal flakes.

Let us consider the same scenario presented in [4]. An automotive radar illuminates a target on the street. The wave reflected by the target will impinge on the printed circuit board (PCB) of the sensor and it will be redirected again towards the bumper. Due to the high permittivity of the bumper, the wave will be reflected back to the sensor. The antennas of the radar will then receive the signal of interest from the target superimposed on the unwanted signal generated by the multiple reflections between sensor and car fascia. Both signals superimpose on the same range cell. The multiple reflections can thus potentially lead to a wrong DoA estimation, which might cause in turn a wrong intervention in the driving process.

Some previous works [4], [5] proposed the use of low-RCS antennas as a possible solution to mitigate the multiple reflections between radar sensor and painted bumper. If the radar is

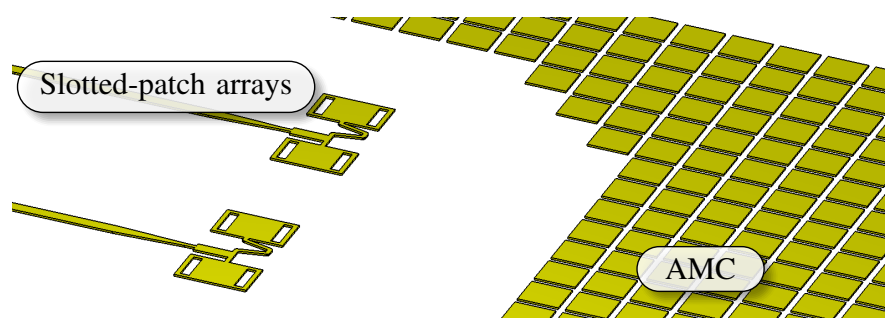

Fig. 1. 3D-model of the low-RCS antenna configuration. A slotted patch array has been integrated with the AMC. Zoomed view of two transceivers.

equipped with such an antenna system, it can scatter back less power in boresight direction towards the bumper, while redirecting the impinging wave towards different directions other than the boresight.

In this work a mm-wave automotive radar that employs a low-RCS MIMO antenna configuration is presented and characterized to demonstrate how the use of low-RCS antennas can effectively improve the angular errors.

\section{Design of The Antenna System}

The principle of operation of the low-RCS antenna employed in this work is based on the chessboard-like surface presented for the first time in [6]. The basic idea is to compensate the backscattering from the antenna by placing an artificial magnetic conductor (AMC) around the radiating element. The AMC should be designed so that destructive interference can be achieved in the wave reflected back by the AMC and the antenna. Following this approach, the antenna arrays developed in [4] and [5] showed a significant RCS reduction compared to the same array without the AMC.

The reduction of the RCS can be formally described by [5]:

$$
r=10 \log \left|w_{\text {AMC }} A_{\text {AMC }} \mathrm{e}^{\mathrm{j} \varphi_{\text {AMC }}}+w_{\text {ant }} A_{\text {ant }} \mathrm{e}^{\mathrm{j} \varphi_{\text {ant }}}\right|^{2} \mathrm{~dB} .
$$

The values $A_{\mathrm{AMC}}$ and $A_{\mathrm{ant}}$ are the amplitudes of the reflection coefficients of the AMC and the antenna array, $\varphi_{\text {AMC }}$ and $\varphi_{\text {ant }}$ are their respective phases. The reflection coefficients can be easily defined, if we assume an impinging plane wave illuminating the AMC and the antenna. The weights $w_{\mathrm{AMC}}$ and $w_{\text {ant }}$ are assigned to the AMC and to the antenna according to their area occupation on the PCB. It is possible to conclude 


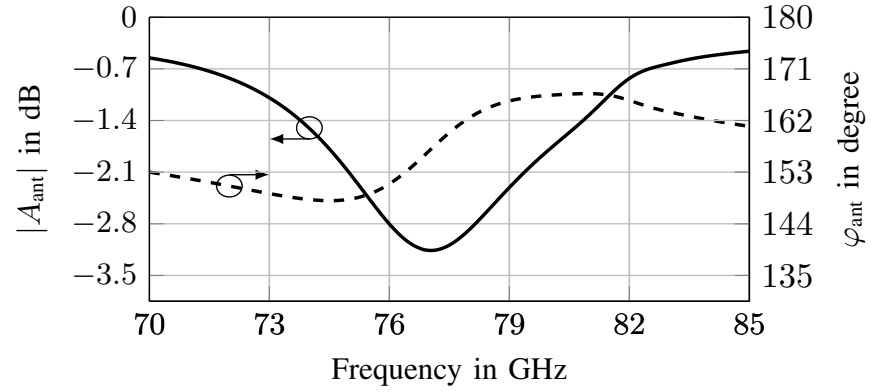

Fig. 2. Full-wave simulation result of the backscattering from the 2-element slotted patch array. Amplitude and phase of the reflection coefficient are depicted in the plot.

then that the equation (1) is a weighted average of the amount of power reflected back by the AMC and the antenna [5].

The model of the low-RCS antenna configuration with the AMC integrated on the array substrate can be seen in Fig. 1. The radar used in this work is an FMCW MIMO system with $M_{t}=3$ transmitting and $M_{r}=4$ receiving elements. The same kind of antenna array is used for both the transmitters and receivers, and it is composed of two slotted patches in parallel, as it can be noticed from Fig. 1. The prototype has been realized with standard PCB-technology on a Teflon-based substrate with a thickness of $127 \mu \mathrm{m}$ and a nominal value of relative dielectric permittivity of 3 .

The first step for the development of the low-RCS array is the evaluation of the backscattering from the antenna system. Due to the complexity of the MIMO configuration, it is not possible to perform a full-wave simulation of the complete antenna system for the calculation of the reflection coefficient required in (1). For this reason, an empirical design approach has been used, similar to the one proposed in [5]. However, while in [5] the terms $A_{\text {ant }}$ and $\varphi_{\text {ant }}$ relative to the whole MIMO configuration were estimated with measurements, in this work the starting point for the design is the simulation of the 2-element slotted-patch array. It is assumed that the reflection coefficient of the whole MIMO antenna system is similar to the one of the 2-element array. Although this solution is not rigorous, the measurement results that will be presented in the next section demonstrate that this method is still useful when alternative approaches, such as analytical solutions or exact full-wave simulations, are not available.

For the simulation of the backscattering from the 2-element patch array, an incident plane wave illuminates the array. An infinite extent of the substrate and of the ground plane has been assumed to neglect the effect of the edges. With this simulation, the reflection coefficient of the antenna that appears in (1), in terms of the amplitude $A_{\text {ant }}$ and of the phase $\varphi_{\text {ant }}$, can be calculated, and it is plotted in Fig. 2 . Afterward, a suitable AMC must be designed so that the RCS can be minimized. In this work the unit cell of the AMC is a squared patched, as it can be seen in Fig. 1. The phase of the AMC must be $180^{\circ}$ out of phase with respect to the one of the array. As it can be noticed from the simulation results of Fig. 3, at $78 \mathrm{GHz}$ the difference between $\varphi_{\text {ant }}$ and

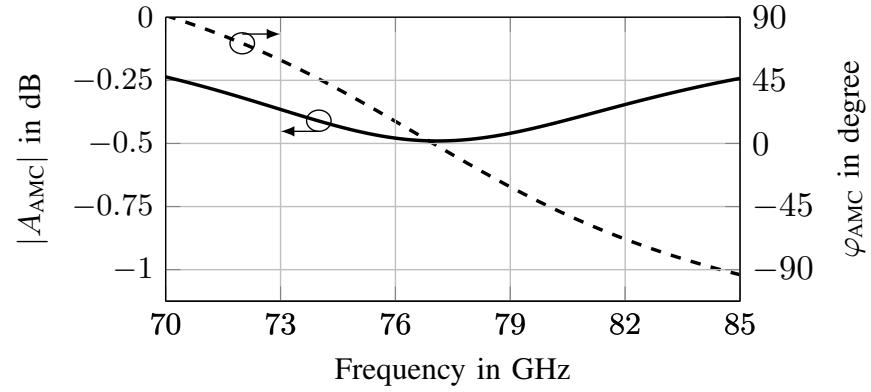

Fig. 3. Full-wave simulation result of the unit cell of the AMC. Amplitude and phase of the reflection coefficient are depicted in the plot.

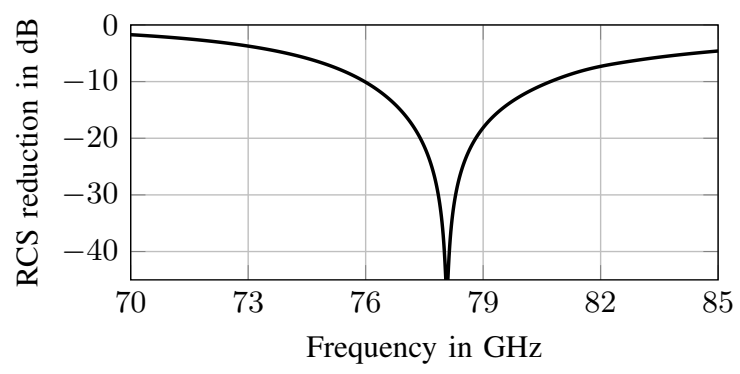

Fig. 4. Calculated RCS reduction using (1)

$\varphi_{\text {AMC }}$ is close to $180^{\circ}$. To take into account the non identical amplitudes $A_{\text {ant }}$ and $A_{\mathrm{AMC}}$, which would not allow a perfect cancellation for a destructive interference, the weights $w_{\text {ant }}$ and $w_{\text {AMC }}$ must be carefully chosen to make $r$ in (1) small, i.e., to reduce the RCS of the antenna system. As it has been already mentioned, the weights describe the area occupied on the PCB by the AMC and the antenna system, respectively. In this case the AMC occupies $43 \%$ of the area and an almost perfect destructive interference can be achieved, as it can be seen from the estimated RCS reduction in Fig. 4.

\section{EXPERIMENTAL CHARACTERIZATION}

A prototype of the proposed MIMO radar equipped with the low-RCS antenna system has been fabricated. To easily evaluate the impact of the low-RCS arrays on the radar performance, a second prototype without the integration of the AMC has been fabricated, too. The MMIC placed on the PCB for the generation of the high-frequency signal has been covered with absorbing material to suppress the spurious radiation that can degrade the radiation pattern. Finally, both prototypes have been covered with a radome. The bandwidth of the FMCW radar is $500 \mathrm{MHz}$ centered around $76.5 \mathrm{GHz}$.

\section{A. Two-Way Radiation Pattern}

The placement of the AMC on the PCB should not affect the radiation patterns of the antennas [4]. For this reason, the two-way pattern for all the 12 virtual transmitter-receiver pairs has been measured. A corner reflector has been placed in the farfield of the sensor, and a sweep on the azimuth plane has been performed. The two-way patterns of the sensors with and without AMC for one channel can be seen in Fig. 5. It can be 


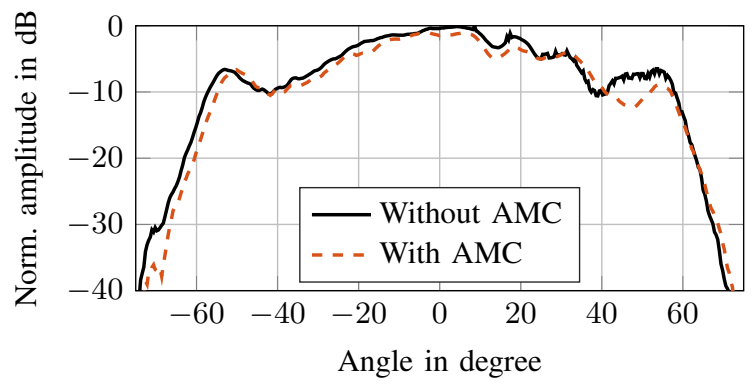

Fig. 5. Normalized two-way radiation pattern using the first transmitter and the first receiver, for both the sensors with and without AMC

noticed that the presence of the AMC does not significantly change the shape of the radiation pattern. Similar results have been obtained for the other channels, and they are here omitted for the sake of brevity.

\section{B. Evaluation of the Angular Error}

The next step in the characterization of the sensor is the measurement behind a sample of painted car bumper made out of polycarbonate with $2.89-\mathrm{mm}$ thickness. With this experimental setup it is possible to evaluate the DoA angular errors due to the presence of the bumper. First, a reference measurement must be taken. It is identical with the measurement of the two-way pattern described previously. Afterward, the bumper plate is placed in front of the sensor, and the two-way pattern is collected again. By comparing the measurements with and without bumper, the angular error can be assessed.

In Section I the problem of the multiple reflections between sensor and bumper has already been extensively described. However, it is important to underline again that, with such an experimental configuration, a standing wave can occur. For a fair evaluation of the measurement results, it is convenient to avoid that the bumper is located exactly at one of the maxima or minima of the standing wave pattern. Therefore, multiple measurements have been collected, where the distance between the sensor and the bumper has been changed in steps of $0.5 \mathrm{~mm}$ starting from the initial value of $10 \mathrm{~mm}$. Moreover, in a practical scenario where the sensor is installed in a car, it is usually hard to exactly control the positioning behind the fascia, typically due to mechanical inaccuracies. Therefore, taking the measurements by slightly varying the distance between the prototype and the bumper sample is a good approach to test also the robustness of the results.

The angular error is calculated using a deterministic maximum likelihood (DML) approach. The mathematical operation that describes the comparison between the measurements with and without the bumper is the correlation. If the correlation is 1 , it means that the two measurements are identical, i.e., the impact of the bumper is negligible. To this purpose, let us define the matrix $\mathbf{C}$, that contains the results of the reference measurement, as follows:

$$
\begin{aligned}
& \text { Ch. } 1 \\
& \text { Ch. } 2 \\
& \vdots \\
& \text { Ch. } M
\end{aligned}\left(\begin{array}{cccc}
\vartheta_{11} & \vartheta_{2} & \ldots & \vartheta_{N} \\
c_{21} & c_{22} & \ldots & c_{1 N} \\
\vdots & \vdots & \ddots & c_{2 N} \\
c_{M 1} & c_{M 2} & \ldots & c_{M N}
\end{array}\right) \text {, }
$$

where $M=M_{t} M_{r}$ is the number of virtual channels and $N$ is the number of sweep points in azimuth. The generic element $c_{i j}$ is a complex value that describes the measurement result at the angular position $\vartheta_{j}$ for the $i$-th virtual channel.

Every element of the matrix can be normalized by dividing it by the norm of the column vector as follows:

$$
\widetilde{c}_{i j}=\frac{c_{i j}}{\left\|\boldsymbol{c}_{j}\right\|},
$$

and to define the matrix $\widetilde{\mathbf{C}}$, which is composed of the elements $\widetilde{c}_{i j}$. In a similar manner, it is possible to build the matrices $\widetilde{\mathbf{A}}$ and $\widetilde{\mathbf{A}}_{\mathrm{AMC}}$, which contain the normalized measurement results of the sensor without and with AMC, respectively, collected after the placement of the bumper in front of the radar. Since the matrices have been already normalized, the cross-correlation can be calculated with the inner product ${ }^{1}$ :

$$
\begin{aligned}
\mathbf{X} & =\left|\widetilde{\mathbf{C}}^{H} \cdot \widetilde{\mathbf{A}}\right|, \\
\mathbf{X}_{\mathrm{AMC}} & =\left|\widetilde{\mathbf{C}}^{H} \cdot \widetilde{\mathbf{A}}_{\mathrm{AMC}}\right|,
\end{aligned}
$$

where the symbol $(\cdot)^{H}$ denotes the complex conjugate (Hermitian) matrix. The two matrices in (4) and (5) describe the crosscorrelation between the two different sets of measurements. For evaluating the angular error, the maximum in every column, i.e., the highest similarity, must be searched. For every column $\boldsymbol{x}_{l}$ in the cross-correlation matrix it is possible to calculate the angular error as

$$
\vartheta_{\mathrm{err}}=\vartheta_{l}-\vartheta_{\mathrm{est}}
$$

where $\vartheta_{\text {est }}$ is defined as

$$
\vartheta_{\text {est }}=\underset{\vartheta}{\arg \max } \boldsymbol{x}_{l}
$$

where the function arg max returns the angular positions where the cross-correlation reaches the maximum.

Figure 6 presents the angular error in the DoA estimation for the two prototypes. The plotted reference value is the autocorrelation of the ideal reference measurement without the bumper, and for this reason it is equal to $0^{\circ}$ in the whole angular range. It is possible to observe that the lowRCS antenna system allows to clearly reduce the error in the DoA estimation in an angular range close to the boresight direction, approximately in the range $\left[-25^{\circ}, 10^{\circ}\right]$. The AMC displacement on the PCB is not symmetric, and thus neither is the pattern of the angular error reduction.

In Fig. 6a for some angular positions a large angular error can be observed, for example at $\vartheta=3.8^{\circ}$ for the $1-\mathrm{mm}$ offset measurement and at $\vartheta=-7.2^{\circ}$ for the 2-mm offset

\footnotetext{
${ }^{1}$ The correlation with zero lag of two signals is equal to their inner product
} 


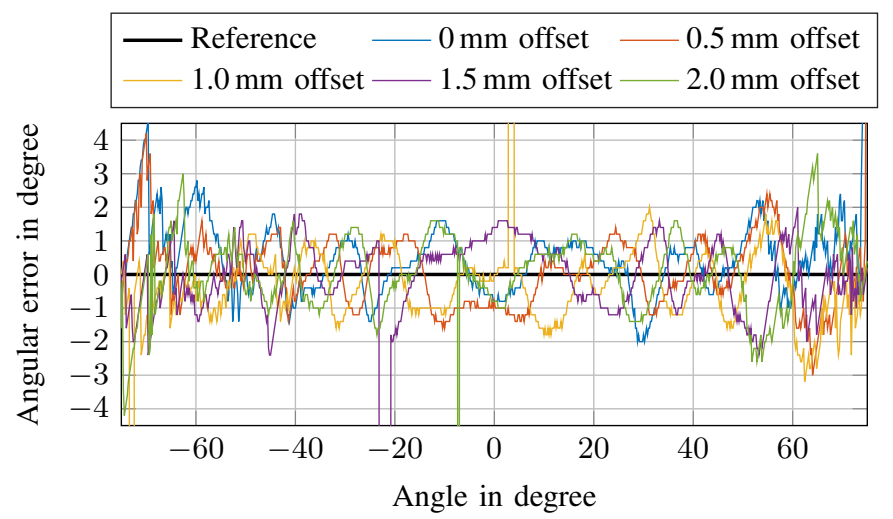

(a) Without AMC

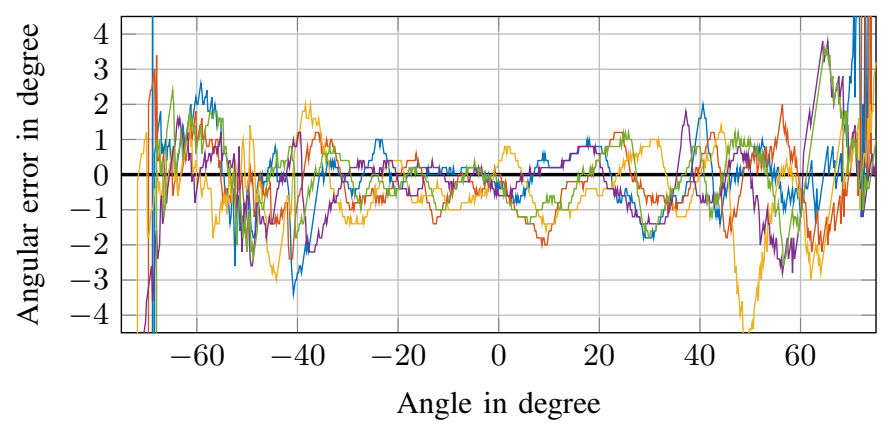

(b) With AMC

Fig. 6. Angular error in the DoA estimation for the prototypes placed behind the bumper. The distance between the prototypes and the bumper has been changed in steps of $0.5 \mathrm{~mm}$. Five different measurements have been collected.

measurement. To explain such results, let us focus on the cross-correlation plots of Fig. 7 for these specific offset and angular values. As it can be understood, large angular errors can be generated by the inaccurate estimation for positions close to the boundaries of the angular range of interest (see Fig. 7a), or by ambiguities such as in Fig. 7b. While the former can be easily avoided by, for example, confining the DoA estimation in a slightly smaller angular range, the latter is a critical issue for the radar sensor, that could be effectively solved by employing low-RCS antennas.

Finally, the standard deviation $\sigma$ of the two sets of measurements has been calculated and plotted in Fig. 8. When the AMC is integrated in the prototype, the value of $\sigma$ is smaller in the range $\left[-25^{\circ}, 10^{\circ}\right]$. It means that the sensor with the lowRCS antennas presents less dispersion from the mean value, and it offers thus an increase in the system robustness.

\section{CONCLUSION}

This paper presented the characterization of a mm-wave radar sensor for automotive applications with a low-RCS antenna system, namely to improve the hidden integration of the radar in a vehicle. The use of low-RCS antennas can mitigate the multiple reflection arising from the placement of the radar behind the car bumper. In particular, the measurement results demonstrated that such a sensor can effectively reduce the angular error in the DoA estimation compared to its counterpart equipped with standard radiating elements.

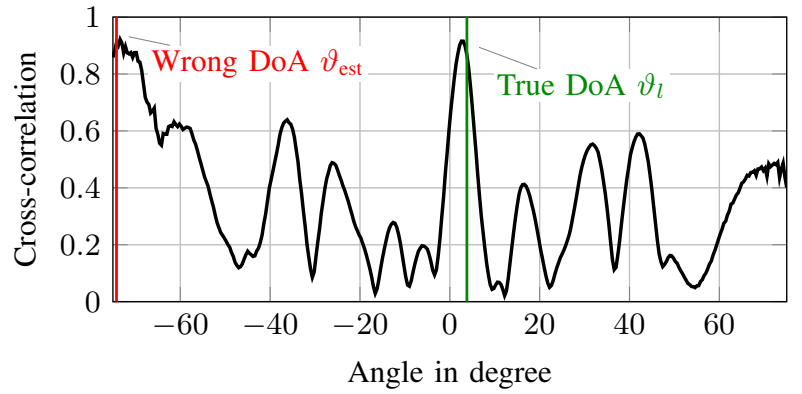

(a) Offset $1 \mathrm{~mm}$

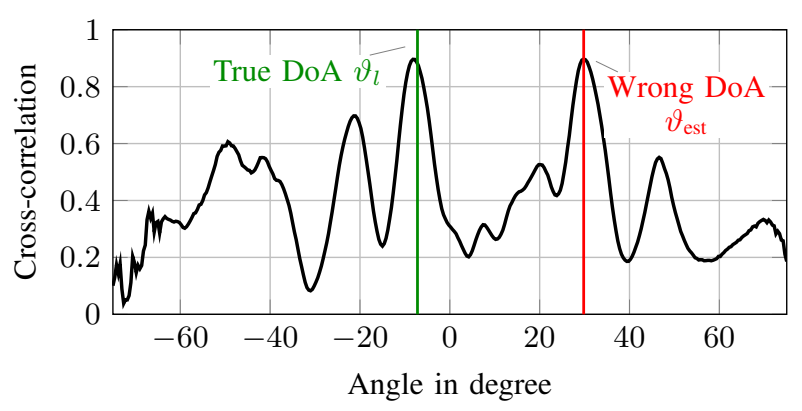

(b) Offset $2 \mathrm{~mm}$

Fig. 7. Cross-correlation of the prototype without the AMC. The wrong DoA of these examples lead to a strong increase in the angular error of Fig. $6 \mathrm{a}$

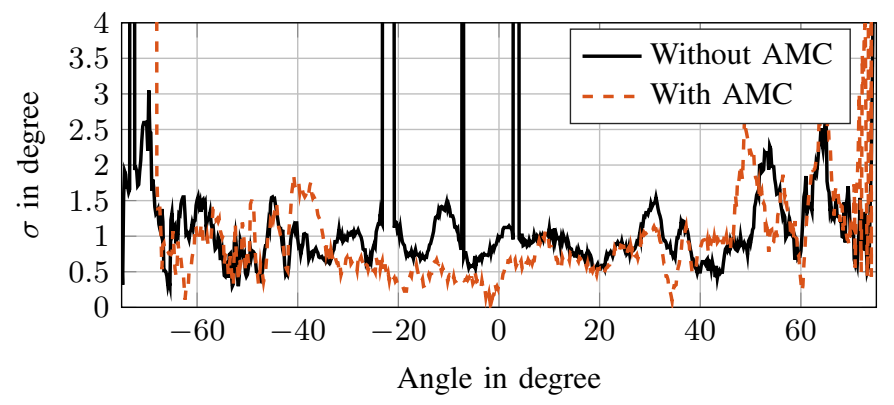

Fig. 8. Standard deviation of the two sets of measurements carried out using the prototypes without and with AMC.

\section{REFERENCES}

[1] W. Menzel and A. Moebius, "Antenna Concepts for Millimeter-Wave Automotive Radar Sensors,” Proc. IEEE, vol. 100, no. 7, pp. 2372-2379, July 2012.

[2] P. Wenig, M. Schoor, O. Gunther, B. Yang, and R. Weigel, "System Design of a $77 \mathrm{GHz}$ Automotive Radar Sensor with Superresolution DOA Estimation," in Int. Symposium Signals, Syst., Electron. IEEE, July 2007.

[3] R. Schnabel, D. Mittelstrab, T. Binzer, C. Waldschmidt, and R. Weigel, "Reflection, Refraction, and Self-Jamming," IEEE Microw. Mag., vol. 13, no. 3, pp. 107-117, 2012.

[4] C. Vasanelli, F. Bögelsack, and C. Waldschmidt, "Reducing the Radar Cross-Section of Microstrip Arrays Using AMC Structures for the Vehicle Integration of Automotive Radars," IEEE Trans. Antennas Propag., vol. 66, no. 3, pp. 1456-1464, March 2018.

[5] C. Vasanelli, F. Bögelsack, and C. Waldschmidt, "A Complex Low-RCS Antenna System for the Placement of mm-Wave Automotive Radars Behind Car Bumpers," in 12th Europ. Conf. Antennas Propag. (EuCAP), London, United Kingdom (Great Britain), 2018.

[6] M. Paquay, J.-C. Iriarte, I. Ederra, R. Gonzalo, and P. de Maagt, "Thin AMC Structure for Radar Cross-Section Reduction," IEEE Trans. Antennas Propag., vol. 55, no. 12, pp. 3630-3638, Dec. 2007. 